\title{
Calculation the Effect of Atomic Number for Projectile and Target on the Electronic Stopping Power
}

\author{
Roaa Salam Kadhum Al- Hasnawei \\ Al Mustqbal College University. Babil, Iraq. \\ ali_s63@yahoo.com
}

Submission date:- 25/11/2018 Acceptance date:- 17/12/2018 Publication date:- 24/2/2019

Keywords: Stopping Power, Distant Collisions, Close Collisions.

\begin{abstract}
In this research, a theoretical study was made to calculate electronic stopping power for heavy charged particles (Protons, Alpha particles, Heavy ions $(\mathrm{C}, \mathrm{O})$ ) which interact with atomic targets $(\mathrm{H}, \mathrm{C}, \mathrm{O}, \mathrm{Si})$ by using the equation which resulting from distant and close collisions, as it has been calculate the effect of atomic number $\left(\mathrm{Z}_{1}\right)$ for projectiles and the effect of atomic number $\left(\mathrm{Z}_{2}\right)$ for targets on the electronic stopping power in range velocities $\left(v_{=}=v_{0}, 2 v_{0}, 5 v_{0}, 10 v_{0}\right)$.
\end{abstract}

A mathematical approximative methods are used in the calculation and some of equation programmed by Matlab language to obtain the theoretical result which are shown in graphic.

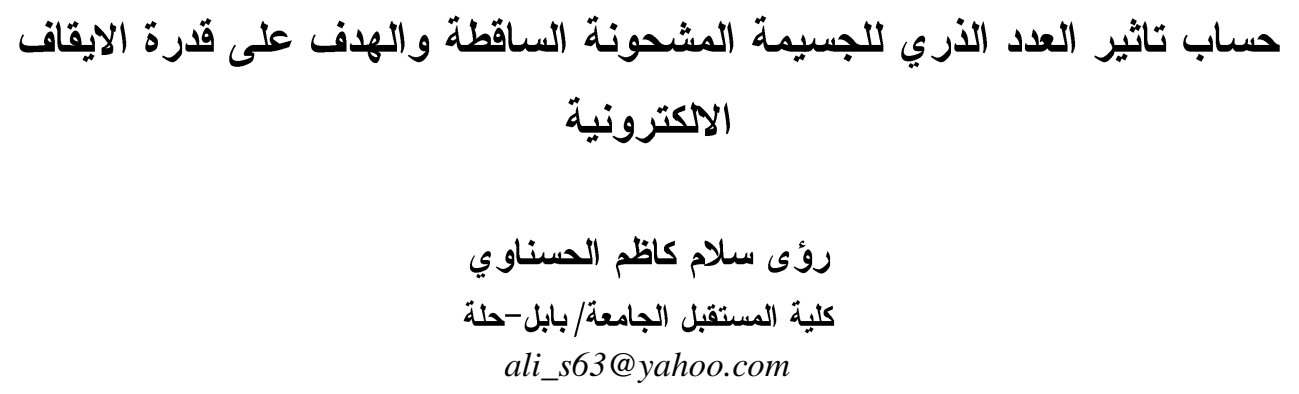

تم في هذا البحث دراسة نظرية لحساب قدرة الايقاف لكل من (البروتونات-جسيمات الفا -ايونات الكاربون - الاوكسجين) المتفاعلة مع

الاهداف (الهيدروجين H و الكاربون C و الاوكسجين O و السليكون Si) وذللك باستخدام المعادلة الناتجة من جمع التصادمات القريبة و البعيدة ،اذ

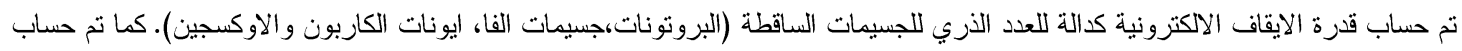

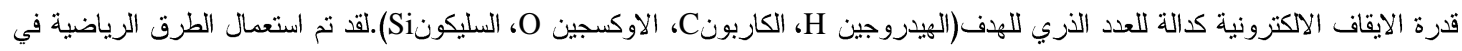

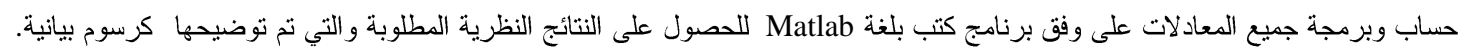

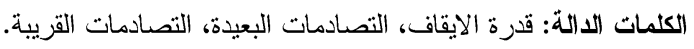

\section{Introduction المقدمة}

عند انتقال الجسيمة المشحونة خلال المادة فأن تفاعلها مع ذرات مادة الهذف ناتج عن القوى الكهرومغناطسية بين الجسيمات المشحونة [1].

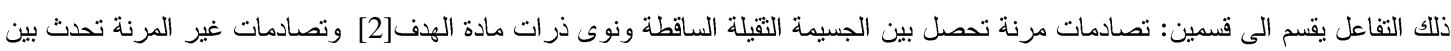

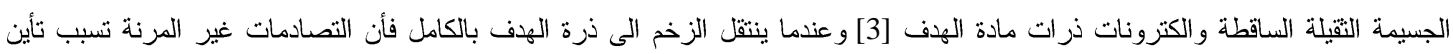

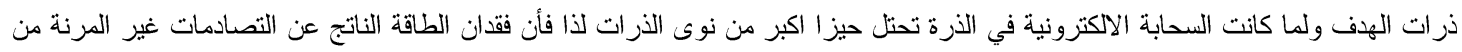


المتوقع ان يكون اكبر من فقدان الطاقة المرن. عند الطاقات الواطئة جدا فأن فقدان الطاقة الكلي الناتج عن التصادمات المرنة يظهر بشكل ملحوظ ويمكن ان يكون بنسبة 15\%-10 من قدرة الايقاف عند 1KeV [5]. سوف ننتاول في هذا الفصل نو عين من التصادمات وهما:

Close Collisions التصادمات القزيبة

Distant Collisions التصادمات البعيدة

\section{Close Collisions التصادمات القريبة 1-1}

لقد تم الافتر اض إن التصادمات القريبة تخضع لقانون استطارة كولوم الحرة (Free Coulomb Scattering) وان حساب فقدان الطاقـــة

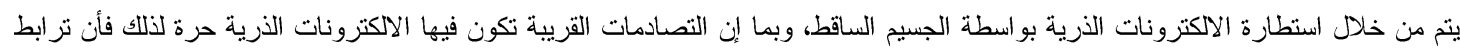

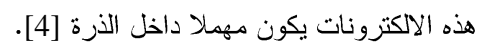

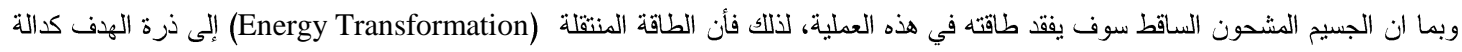

$\mathrm{T}_{\text {close }}=\frac{2 \mathrm{Z}_{1}^{2} \mathrm{e}^{4}}{\mathrm{mv}^{2} \mathbf{p}^{2}} \frac{1}{1+(\mathbf{b} / 2 \mathrm{p})^{2}}$

لمعامل التصادم (p) تعطى بالعلاقة الآتية [6]:

\section{(1)}

TClose هو قطر التصادم (Collision Diameter) ويساوي:

$\mathbf{b}=\frac{2 \mathrm{z}_{1} \mathrm{e}^{2}}{\mathbf{m v}^{2}}$

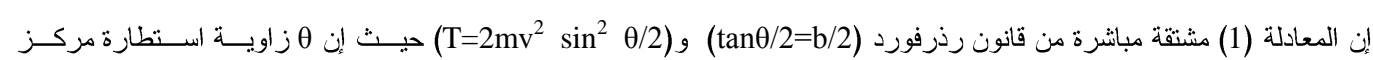

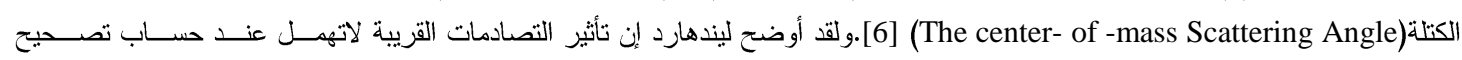

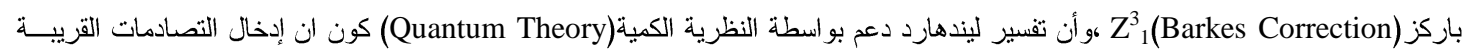

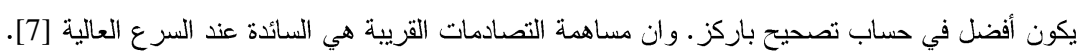

Distant Collisions 2-1 التصادمات البعيدة

ان التصادمات البعيدة تكون فيها الالكترونات الذرية غير حرة، أي أنها ناتجة من تز ابط الكترونات الهانف من خلانل التردد الكلاسيكي

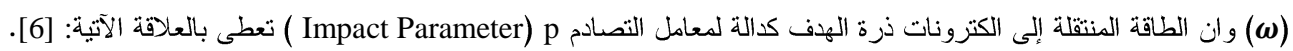

$\mathbf{T}_{\text {dis }}=\frac{2 \mathbf{z}_{1}^{2} \mathbf{e}^{4}}{\mathrm{mv}^{2} \mathbf{p}^{2}}\left\{\left[\mathbf{k}_{0}\left(\frac{\omega \mathrm{p}_{\mathrm{o}}}{\mathrm{v}}\right)\right]^{2}+\left[\mathbf{k}_{1}\left(\frac{\omega \mathrm{p}_{\mathrm{o}}}{\mathrm{v}}\right)\right]^{2}\right\}$

(3)

$$
\text { معامل التصادم الحرج: po }
$$

Modified Bessel Functions ( تمثنل دو ال بيسل المعدلة $\mathbf{k}_{\mathbf{1}} \mathbf{g} \mathbf{k}_{\mathbf{0}}$

و ان مساهمة التصادمات البعيدة في حد باركز تكون هي السائدة في السرع الو الطئة][5].وبما إن قدرة الإيقاف تعرف بدلالة الطاقة المنتقلة

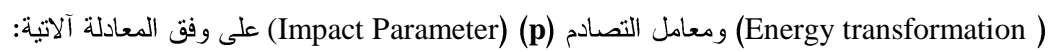

$$
S_{\text {tot }}=\int_{0}^{\infty} 2 \pi T(p) p d p
$$

$S_{\text {tot }}=\int_{0}^{\infty} 2 \pi p d p\left(T_{\text {close }}+T_{\text {dis }}\right)$

$$
=\int_{0}^{\mathrm{po}} 2 \pi p d p \mathrm{~T}_{\text {close }}(\mathrm{p})+\int_{\mathrm{po}}^{\infty} 2 \pi p d p \mathrm{~T}_{\mathrm{dis}}(\mathrm{p})
$$

حيث أن الحد الأول من المعادلة (3-5) يمثل التصادمات القريبة والحد الثاني يمثل التصادمات البعيدة،حيث إن (po (p) هو الذي يحدد

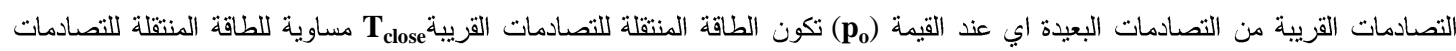
البعيدة Tis

$\mathbf{T}_{\text {close }}\left(\mathbf{p}_{\mathrm{o}}\right)=\mathbf{T}_{\mathrm{dis}}\left(\mathbf{p}_{\mathrm{o}}\right)$

$S_{\text {close }}(p)=\frac{2 \pi Z_{1}^{2} e^{4}}{m v^{2}} \ln \left[1+\left(\frac{2 p_{0}}{b}\right)^{2}\right]$ وبتعويض المعادلتين (1) و (3) في المعادلة (5) نحصل على المعادلتين الآتيتين:

$\mathbf{L}_{\text {close }}=\ln \left[1+\left(\frac{2 p_{0}}{b}\right)^{2}\right]$

$S_{\text {dis }}=\frac{4 \pi \mathrm{z}_{1}^{2} \mathrm{e}^{4}}{m v^{2}}\left(\frac{\omega \mathrm{p}_{\mathrm{o}}}{\mathrm{v}}\right) \mathrm{K}_{0}\left(\frac{\omega \mathrm{p}_{\mathrm{o}}}{\mathrm{v}}\right) \mathrm{K}_{1}\left(\frac{\omega \mathrm{p}_{\mathrm{o}}}{\mathrm{v}}\right)$

$\mathbf{L}_{\text {dis }}=\left(\frac{\omega p_{0}}{v}\right) K_{0}\left(\frac{\omega p_{o}}{v}\right) K_{1}\left(\frac{\omega p_{0}}{v}\right)$ 
$S_{\text {close }}(p)=\frac{2 \pi \mathrm{Z}_{1}^{2} \mathrm{e}^{4}}{m v^{2}} L_{\text {close }}$
$S_{\text {dis }}(p)=\frac{4 \pi \mathrm{Z}_{1}^{2} \mathrm{e}^{4}}{m v^{2}} L_{\text {dis }}$

وبجمع المعادلتين (7) و (9) نحصل على الصيغة العامة لعدد الإيقاف الكلي ( L (L):

$\mathbf{L}_{\text {tot }}=\ln \left[1+\left(\frac{2 p_{0}}{b}\right)^{2}\right]+\left(\frac{\omega p_{o}}{v}\right) K_{0}\left(\frac{\omega p_{0}}{v}\right) K_{1}\left(\frac{\omega p_{0}}{v}\right)$

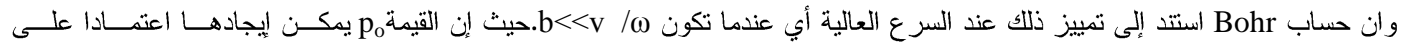

الفترة /

$\mathbf{x k}_{0}(x) \mathbf{k}_{1}(x)=\ln (2 / x)-\gamma+0\left(x^{2}\right)$

$\mathbf{x}=\frac{\omega p_{0}}{\mathbf{v}}$

Y:يمثل ثابت اويلر (Euler's constant) وقيمتة تساوي (0.5772)

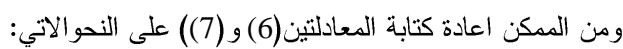

$S_{\text {close }}=\frac{4 \pi \mathrm{Z}_{1}^{2} \mathrm{e}^{4}}{\mathrm{mv}^{2}} \ln \left(\frac{2 \mathrm{p}_{\mathrm{o}}}{\mathrm{b}}\right)$

$\mathbf{L}_{\text {close }}=\ln \left(\frac{2 \mathbf{p}_{\mathrm{o}}}{\mathrm{b}}\right)$

$S_{d i s}=\frac{4 \pi Z_{1}^{2} e^{4}}{m v^{2}} \ln \left(\frac{C v}{\omega p_{o}}\right)$

$\mathbf{L}_{\mathrm{dis}}=\ln \left(\frac{\mathrm{Cv}}{\omega \mathrm{p}_{\mathrm{o}}}\right)$

$S_{\text {tot }}=S_{\text {close }}+S_{\text {dis }}$

[6] حيث ان Cي وبتعويض المعادلات (15) و (17) في المعادلة (19) نحصل على الصيغة العامة لحساب المقطع العرضي للايقاف لنطرية Bohr:

$S_{\text {tot }}=\frac{4 \pi \mathrm{e}^{4} \mathrm{Z}_{1}^{2} \mathrm{Z}_{2}}{\mathrm{mv}^{2}} \ln \left(\frac{\mathrm{Cmv}^{3}}{\mathrm{Z}_{1} \mathrm{e}^{2} \omega}\right)$

$\mathrm{L}_{\text {tot }}=\ln \frac{\mathrm{Cmv}^{3}}{\mathrm{Z}_{1} \mathrm{e}^{2} \omega}$

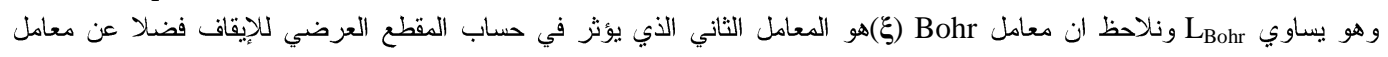

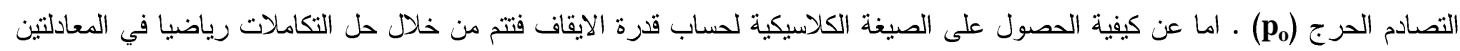

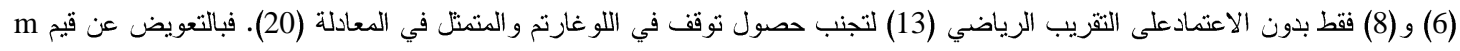

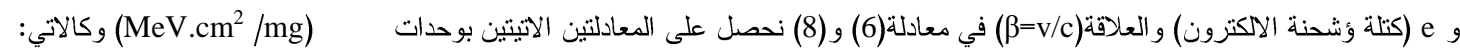
$S_{\text {close }}=0.30707\left(\frac{z_{1}^{2}}{\beta^{2}}\right)\left(\frac{z_{2}}{a_{2}}\right) \times \frac{1}{2} \ln \left(1+\left(\frac{2 p_{o}}{b}\right)^{2}\right)$

$S_{\text {dis }}=0.30707\left(\frac{\mathrm{Z}_{1}^{2}}{\beta^{2}}\right)\left(\frac{\mathrm{Z}_{2}}{\mathrm{a}_{2}}\right)\left(\frac{\omega \mathrm{p}_{\mathrm{o}}}{\mathrm{v}}\right) \mathrm{K}_{\mathbf{0}}\left(\frac{\omega \mathrm{p}_{\mathrm{o}}}{\mathrm{v}}\right) \mathrm{K}_{1}\left(\frac{\omega \mathrm{p}_{\mathrm{o}}}{\mathrm{v}}\right)$

وعند جمع هاتين المعادلتين نحصل على المعادلة الكلاسيكية العامة لحساب المقطع العرضي للايقاف التي تم الاعتماد عليها في حساباتتا

$S_{\text {tot }}=0.30707\left(\frac{z_{1}^{2}}{\beta^{2}}\right)\left(\frac{Z_{2}}{a_{2}}\right) L_{\text {tot }}$

$\mathbf{L}_{\text {tot }}=\frac{1}{2} \ln \left(1+\left(\frac{2 p_{0}}{b}\right)^{2}\right)+x_{0}(x) K_{1}(x)$

عدد الايقاف الكلي للمعادلة (24) يعطى بالعلاقة الاتية:

- a

The effective parameters for calculating the stopping cross section من المعادلتين (6) و (8) و الناتجة منها معادلة Bohr (20) نلاحظ ان هناك معاملات اساسية تؤثز في حساب المقطع العرضي

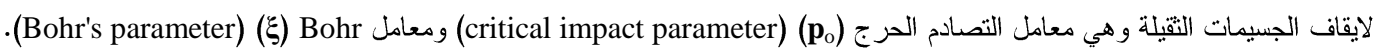

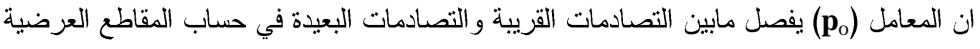

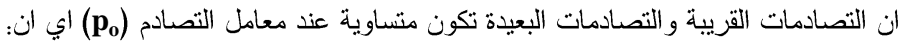

$S_{\text {close }}=S_{\text {dis }}$ 
$\ln \left(\frac{C v}{\omega p_{o}}\right)=\ln \left(\frac{2 p_{0}}{b}\right)$

$$
\therefore \mathbf{p}_{\mathbf{0}}=\left(\left(\frac{\mathrm{Cvb}}{2 \omega}\right)^{\frac{1}{2}}\right)
$$

$p_{o}=\left(\frac{C Z_{1} \mathrm{e}^{4}}{\mathrm{mvv}_{0} \mathrm{I}}\right)^{1 / 2}$
فمن مساو اة المعادلة (15) و(17) نحصل على:

وبالتعويض عن ( I/h) و المعادلة (2) في المعادلة (28) نحصل على:

\section{2-1-ابحسابات و النتائج \\ -1-2 حساب تاثير العدد الذري للجسيمة المشحونة الساقطة والهاف على قدرة الايقاف الاكترونية} Calculation effect the atomic number for projectile and target on the electronic stopping power

لقد تم حساب قدرة الايقاف الآكترونية لكل من البروتونات وجسيمات الفا وايونات الكاربون والاوكسجين ذات العدد الذري

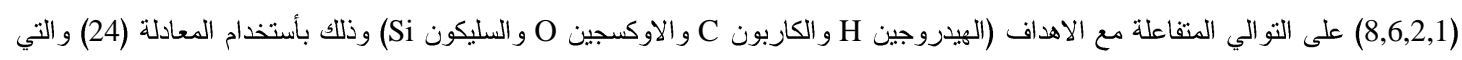
تمت برمجتها باستخدام برنامج بلغة Matlab والتي اعتمدناها في حساباتتا النظرية اذ تم حساب قدرة الايقاف الألكترونية كدالة للعدد الذربي للجسيمات الساقطة كما في الثكل (1) الذي يوضح مجموعة من الجسيمات الساقطة (P,Alpha particle,C,O) على الاهداف الذرية على التو الي. كما تم حساب قدرة الايقاف الالكترونية (P,C,O,Si) موضح في الثكل (2) عند السرع (v=vo,ve2vo, الساقطة عند السرع (v=vo,v=2

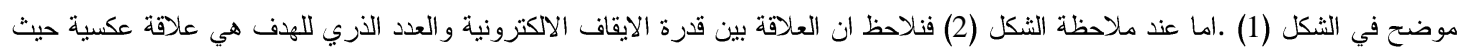

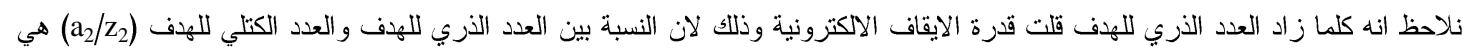

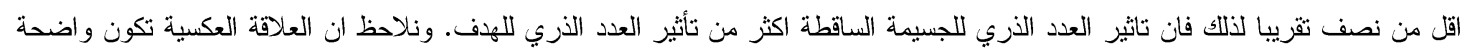
اكثز لاسيما عندما تكون السرعة (v=v).

$\mathrm{P}+$ Alpha particle+C+O Ion $\mathrm{NNH}$

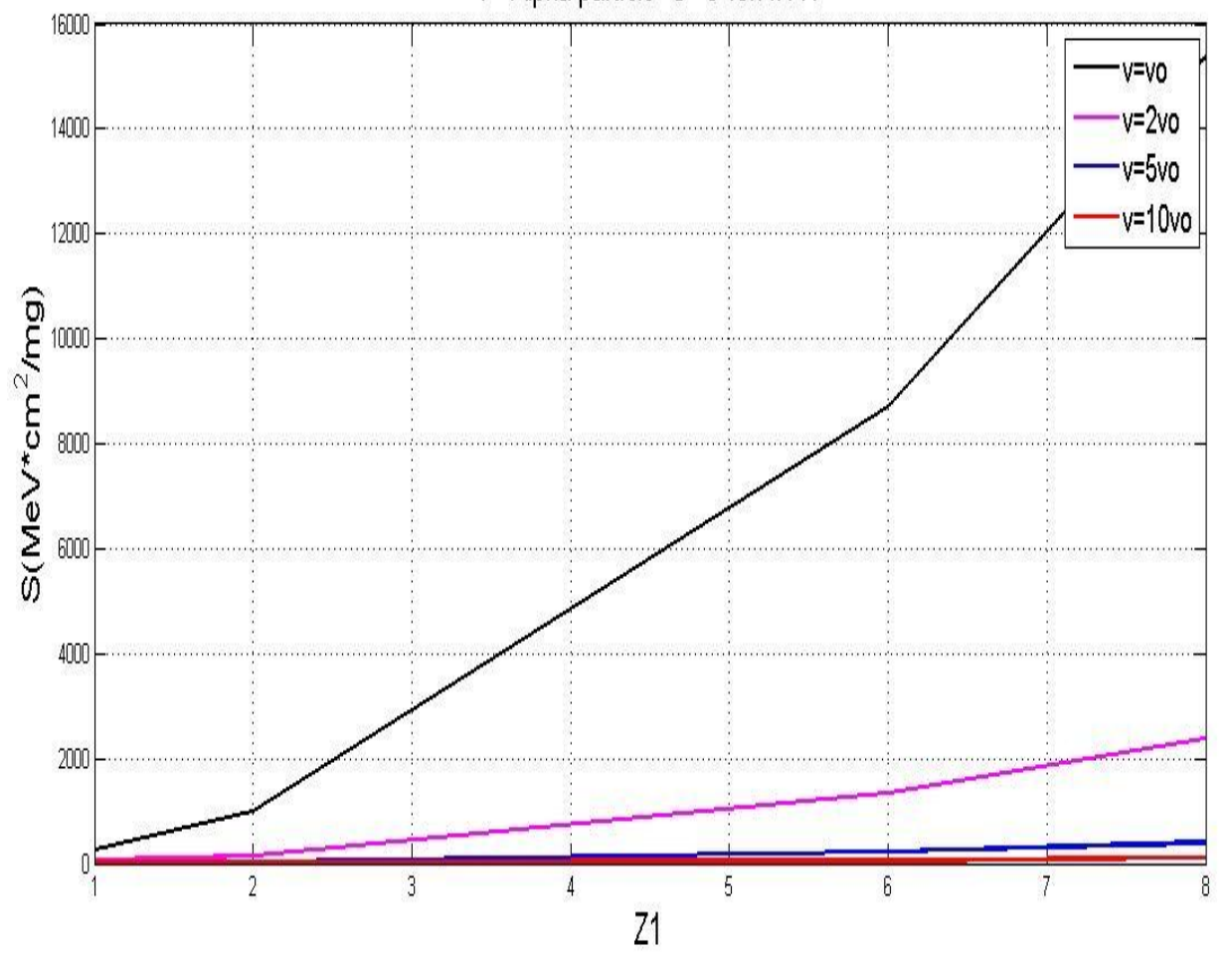

(a) P+Alpha particle+C+O IN $\mathrm{H}$ 


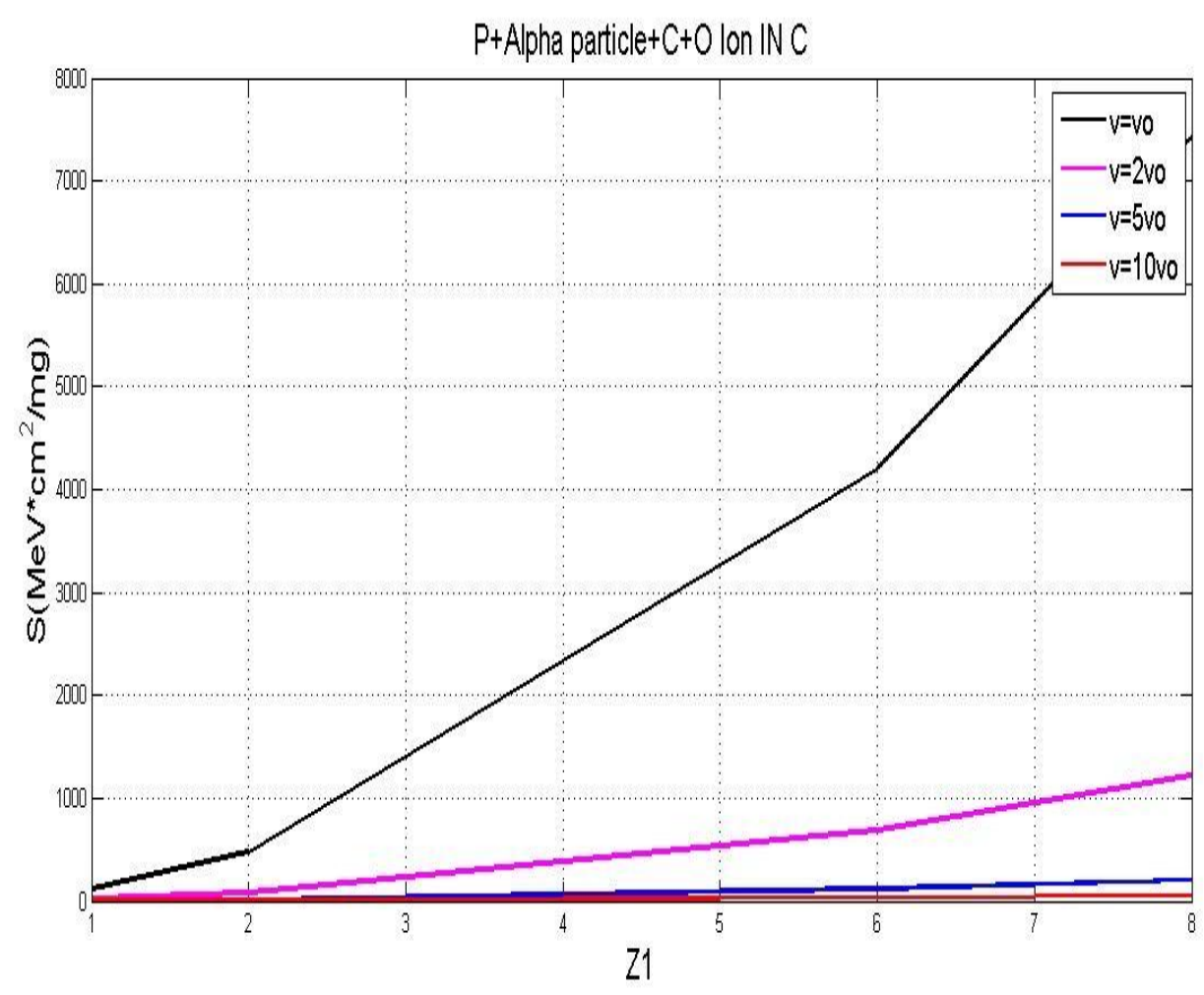

(b) $\mathrm{P}+$ Alpha particle+C+O IN C

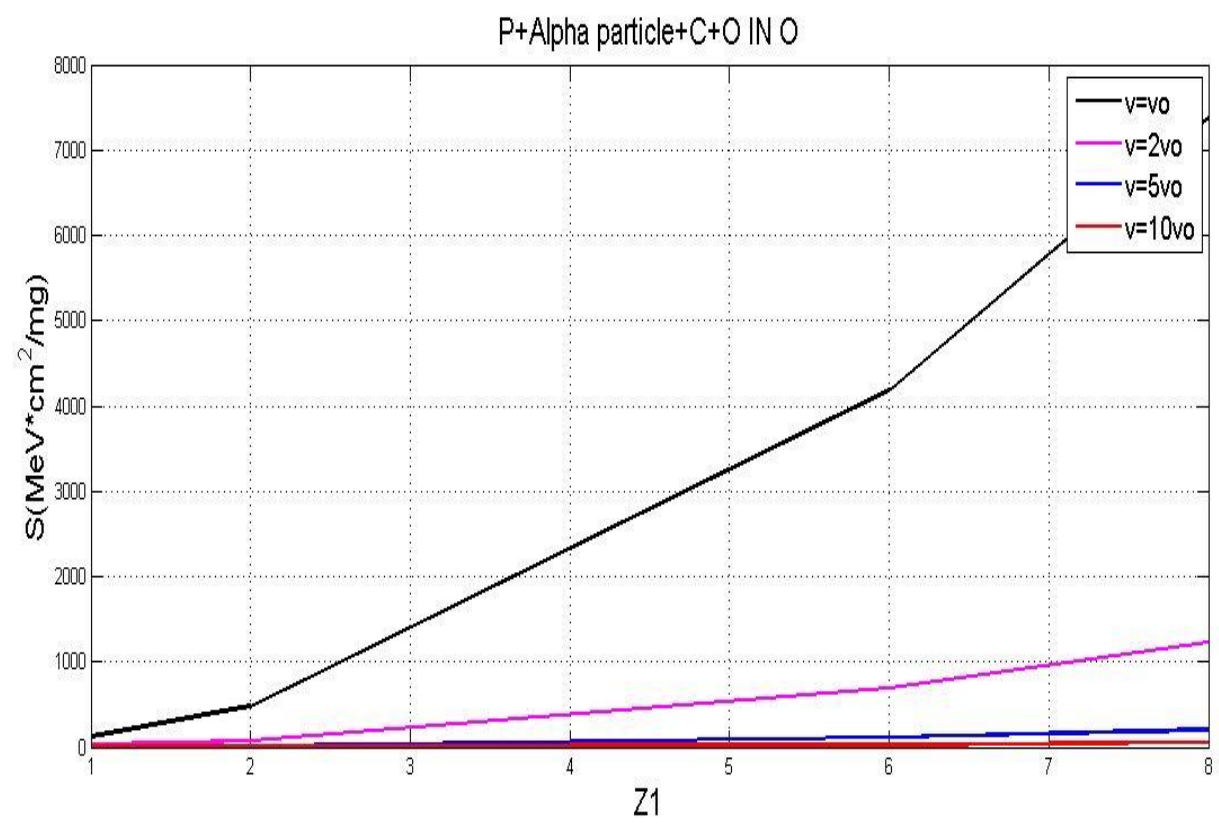

(c) P+Alpha particle+C+O IN O 


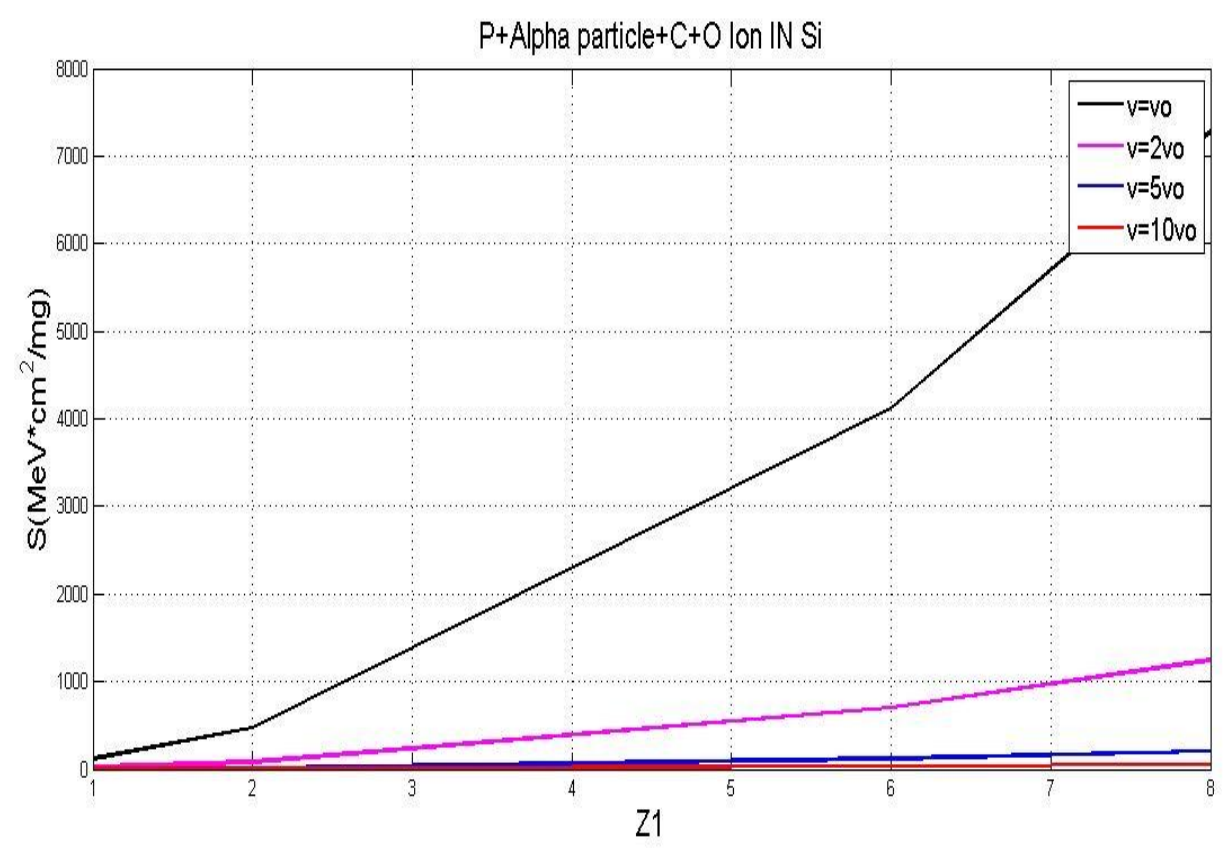

(d) P+Alpha particle+C+O IN Si

الثكل (1) تاثير العدد الذري للجسيمات الساقطة على الاهداف الذرية (Si,O,C,H) باستخدام المعادلة الناتجة من تاثير التصادمات القريبة

$\mathrm{P}|\mathrm{N} \mathrm{H}+\mathrm{C}+\mathrm{O}+\mathrm{S}|$

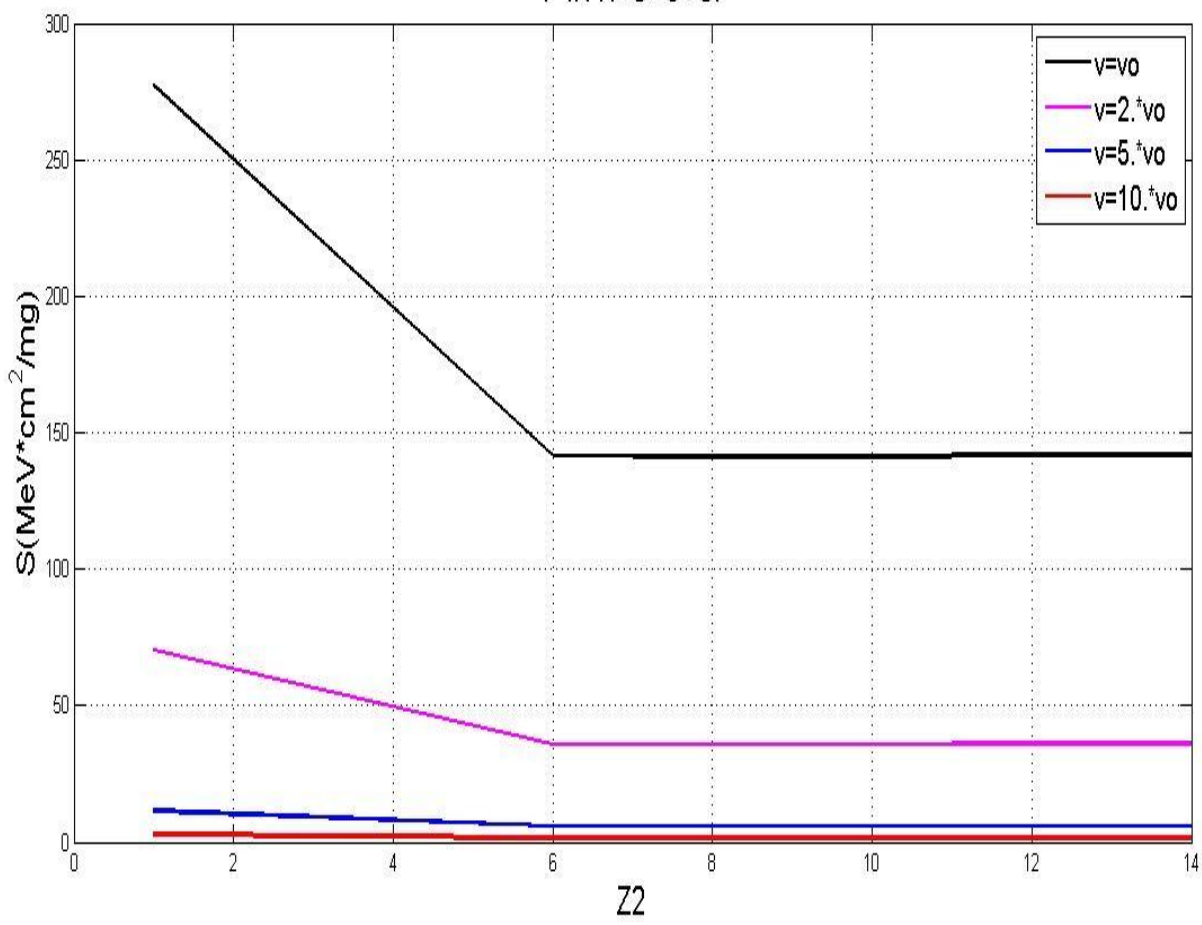

(a) $\mathrm{P}+\mathrm{IN} \mathrm{H}+\mathrm{C}+\mathrm{O}+\mathrm{Si}$ 


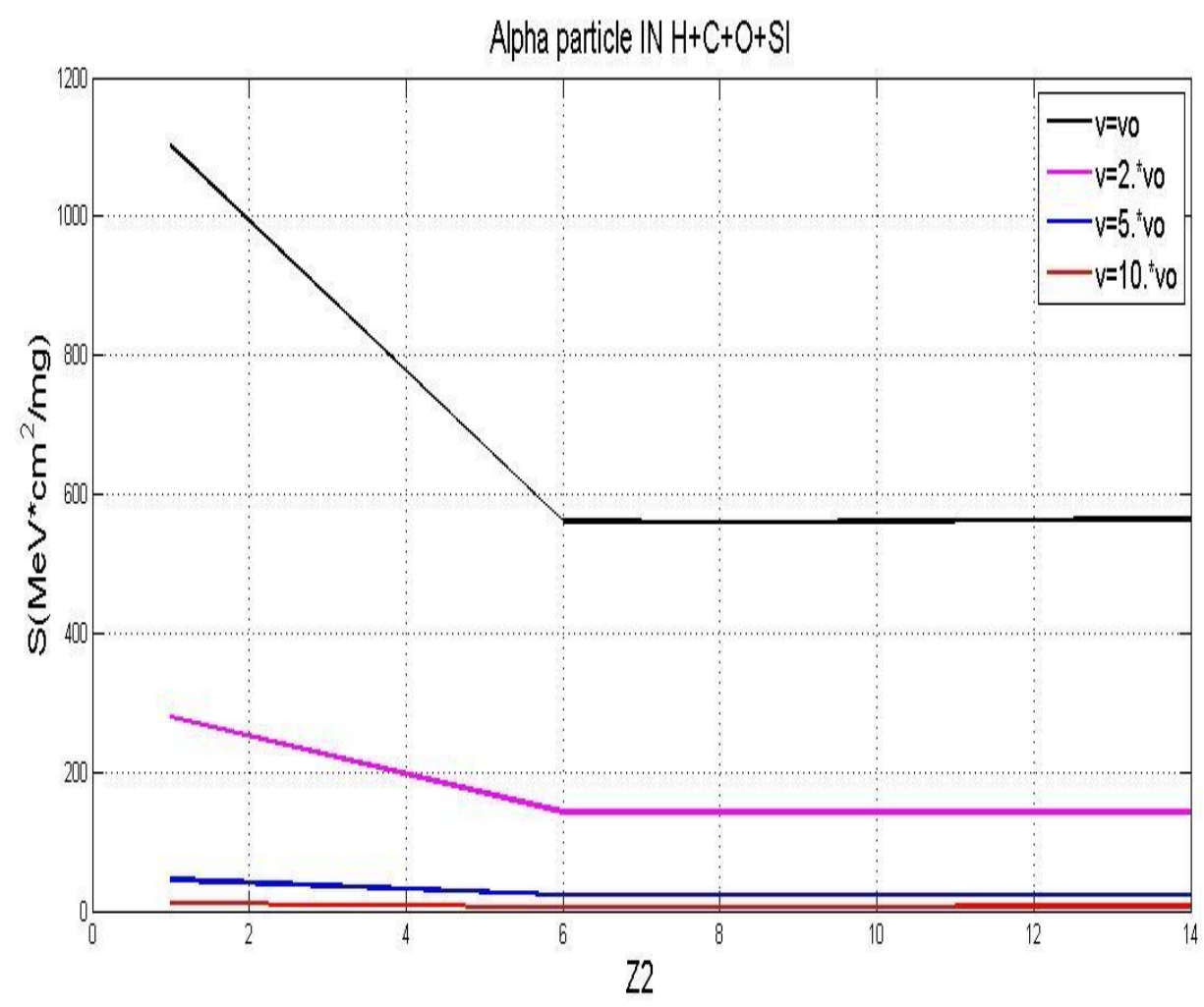

(b) Alpha particle IN $\mathrm{H}+\mathrm{C}+\mathrm{O}+\mathrm{Si}$

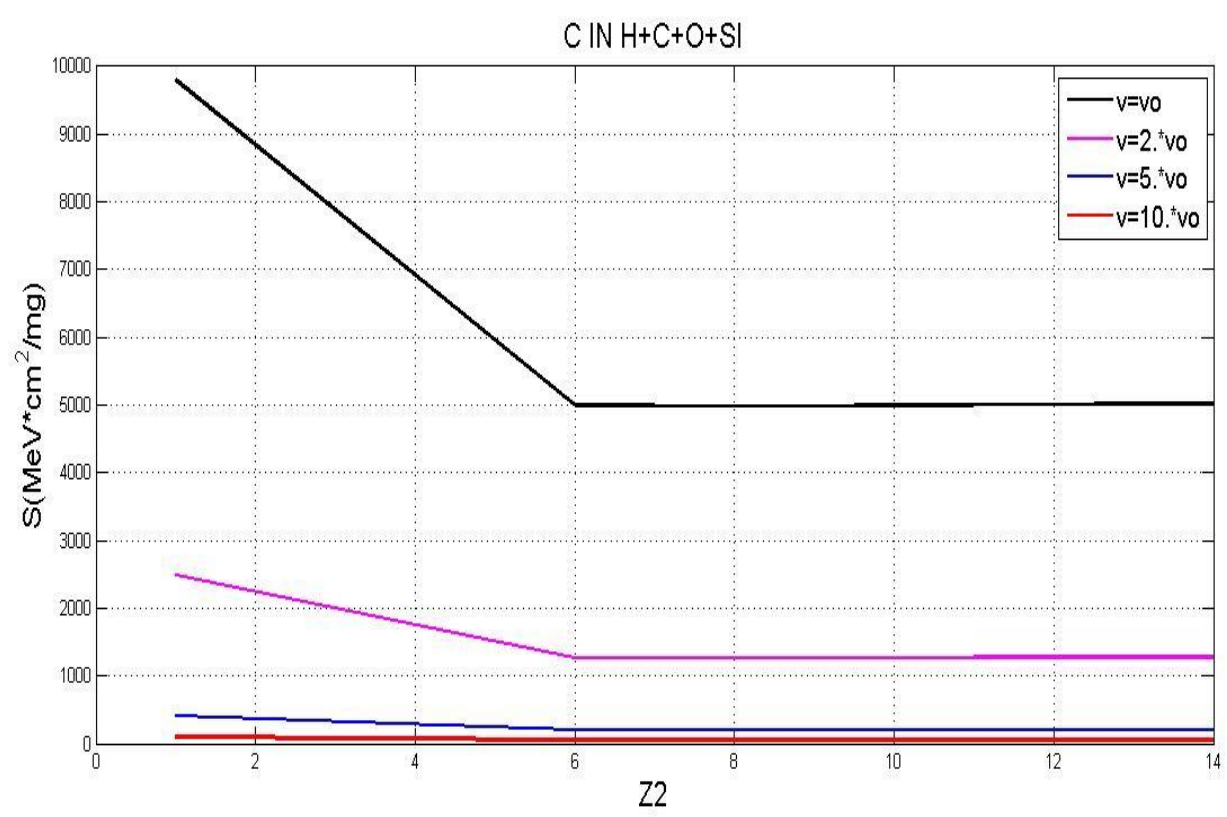

(c) $\mathrm{C}$ IN $\mathrm{H}+\mathrm{C}+\mathrm{O}+\mathrm{Si}$ 


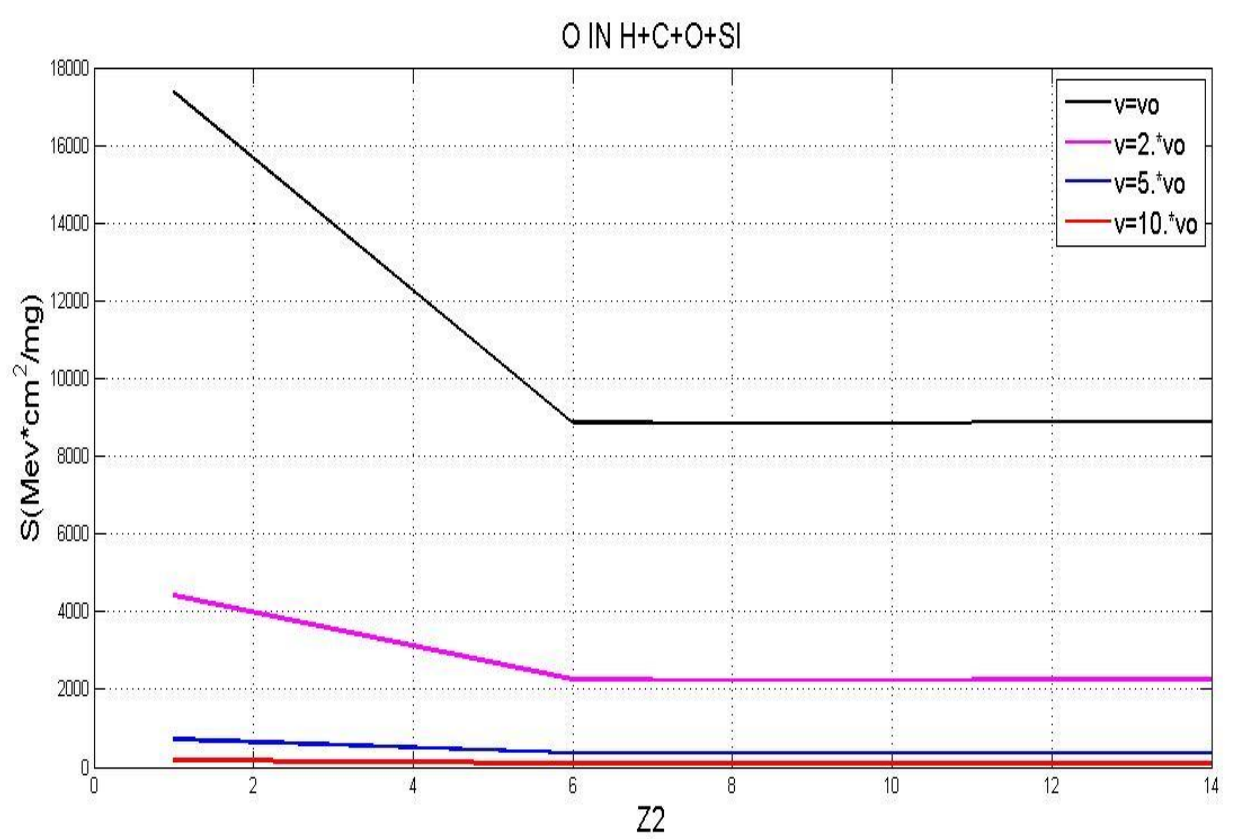

(d) $\mathrm{O}$ IN $\mathrm{H}+\mathrm{C}+\mathrm{O}+\mathrm{Si}$

الثكل (2) يوضح تاثير العدد الذري للاهداف الذرية (Si,O,C,H) على قدرة الايقاف الاككترونية باستخدام المعادلة الناتجة من تاثير

r-الاستنتاجات

من خلال دراستتا لقدرة الايقاف الالكترونية يمكن ان نستتتج النقاط المهمة الاتية:

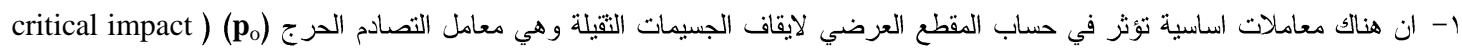

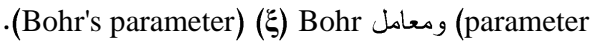
r- يمكن استخدام المعادلة (24) في حساب قدرة الايقاف الاكترونية لكل من البروتو نات وجسيمات الفا و ايونات الكاربون والاوكسجين ذات العدد

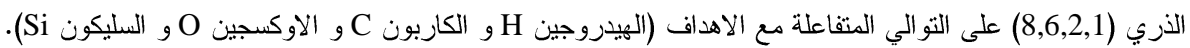

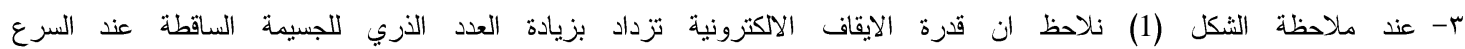

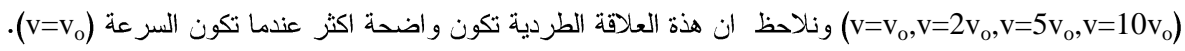

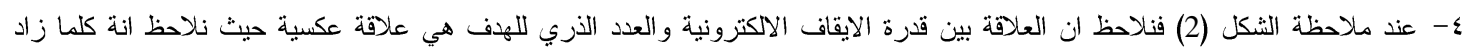

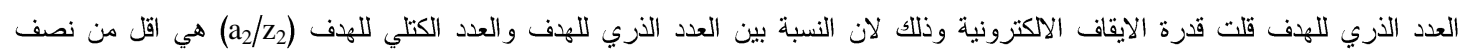

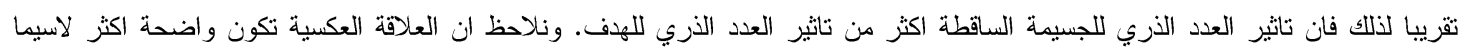

\section{CONFLICT OF INTERESTS}

عندما تكون السرعة (v=volo

\section{There are no conflicts of interest.}

References المصادر

[1]Csete A.,"Experimental investigations of the energy loss of slow protons and antiprotons in matter",M.Sc.Thesis,Instute of physics and astronomy,University of Aarhus,2002.

[2]Meyerhof W.E.," Elements of Nuclear Physics", New York, McGraw-Hill,1967.

[3]Evan R.D., "The Atomic Nucleus" ,New York, ,McGraw-Hill, 1955.

[4]Aziz.Al-Rubyi,"Increase the range of stopping power of energies

$(1<\mathrm{E}(\mathrm{MeV} / \mathrm{u}) \leq 0.1)^{\prime \prime}, \mathrm{M} . S c . T h e s i s, \mathrm{Al}-$ Mustansiriyah university,1999.

[5] Ziegler J.F. and J.P. Biersack, [SRIM.com] contribution byM.Ziegler ,D.J.Marwick,G.A.Cuomo,W.A.Porter,S.A.Harrison [SRIM-2003.12].

[6]Sigmund P., ,"Low-speed limit of Bohr's stopping - power formula",Phys.Rev A.Vol.54,No.4,1996,

[7]Najem K.S.,"Shell and inverse Bloch correction computing from stopping power theories of Bohr and Bethe",M.Sc.Thesis,Al- Mustansiriyah university,2010. 